dilution, storage, administration and stability was observed, when considering the different formulations of the same active substance, which depend on the manufacturer. In all, 32 manufacturers were asked to add additional relevant information that was not present in the SPC. The guide is available in electronic format and in A5 print format (handbook), which has proved to be very practical, fast and effective to use.

Conclusions The published guide is a valuable tool for all Portuguese hospital pharmacists who prepare parenterally administered chemotherapy, answering to most information needs on reconstitution, dilution, storage, stability and administration of injectable cytotoxic drugs.

No conflict of interest.

\section{DGI-040 HUMAN LUNG CARCINOMA SENSITIVITY TO PACLITAXEL: WHICH ROLE FOR BIM?}

doi:10.1136/ejhpharm-2013-000276.306

II Pobel, ${ }^{1} \mathrm{~A}$ Savry, ${ }^{1} \mathrm{M}$ Le Grand, ${ }^{2} \mathrm{~V}$ Rey, ${ }^{3} \mathrm{D}$ Braguer. ${ }^{1}$ Assistance publique - Hôpitaux de Marseille, Marseille, France, ${ }^{2}$ 29112Inserm U - CRO, Marseille, France, ${ }^{39112 I n s e r m ~ U ~}$ CRO, Marseille, France

Background Deregulation of apoptosis is one of the causes of cancer developing. The Bcl-2 family are central regulators of apoptosis. They are subdivided into two classes, the proapoptotic members (which include Bim) and antiapoptotic members (like Bcl-2). The overexpression of $\mathrm{Bcl}-2$ is generally associated with many cancers and resistance to chemotherapy, including microtubule-targeting agents (MTAs). Therefore several anti-Bcl-2 strategies are in development. Unexpectedly, several studies show that a decrease in Bcl-2 may be associated with resistance to MTAs. This paradoxical role of $\mathrm{Bcl}-2$ has not yet found a clear explanation.

Purpose To show that overexpression of Bcl-2 leads to overexpression of Bim, which is responsible for increasing sensitivity to MTAs. Bim is a potential biomarker which may be included in tests to predict the response to paclitaxel treatment in human lung carcinoma. Our work also enables a better understanding of how Bim regulates genes. Materials and Methods The techniques used to study the sensitivity of cells to MTAs are the Western Blot and immunofluorescence. To study Bim's regulation of genes, we used the technique of a reporter gene.

Results Firstly, we showed that overexpression of Bcl-2 in human lung carcinoma cells (A549 Bcl-2) in turn triggers the overexpression of Bim. Apoptosis is detected after treatment with paclitaxel at $20 \mathrm{nM}$, after 24 hours. For this, we used the anti-caspase 9 antibody to show it was being cleaved and to signal the release of the apoptosis mitochondrial pathway. To confirm this, we used immunofluorescence staining to objectify the release of cytochrome $\mathrm{c}$ from the mitochondria. So we showed that the overexpression of Bim in cells that overexpress $\mathrm{Bcl}-2$ accounts for their increased sensitivity to paclitaxel.

We also conducted a study of gene regulation by Bim in A549 cells overexpressing Bcl-2. We highlighted the increasing transcriptional activity of Bim promoter by a factor of $2.3 \pm 0.2$ compared to control cells. The Bim protein level seems to be a better determinant of MTAs sensitivity than Bcl-2 status in pulmonary epithelial tumours. Thus, it appears that Bim expression may be an effective biomarker in predicting the efficiency of MTA treatment. We are currently evaluating the involvement of various transcription factors, especially by DNA microarray.

Conclusions These data suggest that Bim is a more reliable marker of the sensitivity to MTAs than Bcl-2. A test showing the level of Bim expression may be able to predict therapeutic efficacy and/or resistance based on molecular profiling of the tumours. However, the induction of Bim alone cannot be sufficient for significant cell death. Indeed, it is more likely that Bim acts in unison with the other pro-apoptotic proteins. So the development of targeted therapies, on the Bcl-2 family in particular, must await a better understanding of the molecular mechanism involved in the regulation of apoptosis.

No conflict of interest.

\section{DGI-041 HYPOMAGNESEMIA AS A POSSIBLE MARKER OF EFFECTIVENESS IN PATIENTS TREATED WITH PANITUMUMAB}

doi:10.1136/ejhpharm-2013-000276.307

J Megías Vericat, J Ruiz Ramos, J Reig Aguado, C Borrell García, MJ Esteban Mensua, E López Briz, JL Poveda Andrés. Hospital Universitario La Fe, Servicio de Farmacia, Valencia, Spain

Background Panitumumab is a human monoclonal antibody indicated in the treatment of colorectal carcinoma (CRC) that is currently being tested in otolaryngology (ENT) tumours. Recent studies suggest that hypomagnesaemia $(<1.7 \mathrm{mg} / \mathrm{dL})$ during treatment with panitumumab may be related to greater anti-tumour efficacy.

Purpose To review the effectiveness of panitumumab and its possible relationship with hypomagnesaemia

Materials and Methods Retrospective observational study that included all patients treated with panitumumab in a tertiary hospital. The primary endpoint of effectiveness was overall survival (OS), calculated using the Kaplan Meier method. We examined anthropometric data, diagnosis, treatment duration and, in patients whose magnesium had been determined during panitumumab treatment, we also studied the causes of termination and adverse reactions.

Results During the study period (August 2008-October 2012) 72 patients were treated, who had an average baseline age of 63 (SD:11) years and were mostly male (56\%). At the end of the study $47 \%$ of patients were alive and $44 \%$ of them are still being treated with panitumumab. Diagnosis of $89 \%$ of patients was CRC, while 8 ENT cancer patients were enrolled in a clinical trial. The average length of treatment was 4.9 (SD:5.7) months and 7.7 (SD:6.4) cycles/ patient were administered.

Magnesium levels were only determined in 13 patients, hypomagnesaemia being detected in 6 patients (ENT:3, CCR:3) and normomagnesaemia in the remaining 7 (ENT:5, CCR:2). Treatment with panitumumab was stopped in 6 patients due to disease progression. Two patients had to reduce the dose due to severe skin toxicity. The OS was calculated in all patients [hypomagnesaemia: 9.5 (95CI:4.9-14.0) vs. normomagnesaemia 8.2 (95CI:4.2-12.3) months (p:0.703)] and in the ENT tumours subgroup [hypomagnesaemia: 13 (95CI:9.3-16.7) vs. normomagnesaemia 4.8 (95CI:2.96.8) months (p:0.127)].

Conclusions Despite the low magnesium determinations we observed a trend to greater $O S$ in hypomagnesaemic patients. Further studies are needed to confirm this trend.

Abstract DGI-041 Table 1

\begin{tabular}{lllll}
\hline & $\mathbf{n}$ & Dead/alive & OS (95CI) & $\mathbf{p}$ \\
\hline All patients & 72 & $38 / 34$ & $17.0(13.2-20.7)$ & \\
CRC & 64 & $34 / 30$ & $17.0(13.1-20.9)$ & 0.952 \\
ENT & 8 & $4 / 4$ & $9.3(5.2-13.3)$ & \\
\hline
\end{tabular}

No conflict of interest.

\section{DGI-042 HYPOMAGNESEMIA AS A POSSIBLE MARKER OF EFFICACY IN PATIENTS WITH HEAD AND NECK CARCINOMA IN FIRST-LINE TREATMENT WITH CETUXIMAB}

doi:10.1136/ejhpharm-2013-000276.308

J Ruiz, JE Megías, C Borrell, P Marrero, E López, JL Poveda. ${ }^{1}$ Hospital Universitario La Fe, Pharmacy, Valencia, Spain 
Background It has been reported that the determination of magnesium levels could be used as a surrogate marker of efficacy in chemotherapy regimens with cetuximab.

Purpose To investigate the hypomagnesemia caused by cetuximab as a predictor of efficacy and outcome in patients affected by head and neck cancer in first-line treatment.

Materials and Methods Retrospective observational study (Study period: November 2008-October 2012). We analysed patients with head and neck carcinoma treated with cetuximab in first-line treatment, who had magnesium determinations from the start of treatment until one month after the end of treatment with cetuximab. Patients with magnesium determinations were stratified into two groups: Patients who presented hypomagnesemia during the treatment $(<1.7 \mathrm{mg} / \mathrm{dL})$ and patients who didn't present hypomagnesemia. The primary outcome was to compare remission rate, progression-free survival (PFS) and overall survival (OS) in the two groups. PFS and OS were both determined by the Kaplan-Meier product-limit method.

Results We collected a total of 14 patients (92.8\% male). The median age at onset of treatment was 61 years (range: $21-86$ ). Six patients developed hypomagnesemia during treatment. The most common diagnosis was carcinoma of the oral cavity $(28.6 \%)$ followed by laryngeal carcinoma (21.4\%). The group of patients who presented hypomagnesemia showed a higher remission rate $(66.7 \%$ vs. $37.5 \%$ patients), OS [mean: 34.8 (18.8 to 50.9$)$ ) vs. 22.4 (95\% CI: 11.9 to 32.9 months, $p=0.532$ ] and PFS [34.5 months (18.11 to 50.9), vs. 19.7 (7.8-31.5) $\mathrm{p}=0.456$ ] in comparison with the group in which hypomagnesaemia was not detected.

Conclusions Despite the small number of patients studied, hypomagnesemia could be a marker of cetuximab efficacy in first-line treatment in patients with head and neck cancer. Magnesium levels should be determined routinely in patients treated with cetuximab.

No conflict of interest.

\section{DGI-043 INCREASED COST OF ERYTHROPOIESIS-STIMULATING AGENTS IN SOME SPECIAL SITUATIONS}

doi:10.1136/ejhpharm-2013-000276.309

'V Escudero-Vilaplana, 2JM López-Gómez, ${ }^{2} \mathrm{~A}$ Vega-Martínez, 'A Ais-Larisgoitia, 'B Marzal-Alfaro, 'I Yeste-Gómez, 'A Ribed-Sánchez, 'P Arrabal-Durán, 'A GiménezManzorro, 'M Sanjurjo-Sáez. 'Hospital General Universitario Gregorio Marañón, Pharmacy, Madrid, Spain; ${ }^{2 H}$ ospital General Universitario Gregorio Marañón, Nephrology, Madrid, Spain

Background Situations such as a previous kidney transplant or dialysis may increase the dose requirement of erythropoiesisstimulating agents (ESAs), which is associated with a higher cost.

Purpose To examine the difference in cost between the use of ESAs for pre-dialysis and dialysis (peritoneal dialysis or haemodialysis) patients and for patients with or without a kidney transplant.

Materials and Methods A descriptive, transversal study was carried out in patients treated with ESAs for anaemia secondary to chronic kidney disease in a tertiary hospital over a month in 2011. ESAs used were: epoetin ( $\alpha$ or $\beta$ ), darbepoetin $\alpha$ and continuous erythropoietin receptor activator (CERA). The principal variable was patient-month cost, calculated as the cost of the dose unit for each ESA type (IU or mcg) multiplied by the monthly dose per patient.

Results 333 patients were included. $26.2 \%$ had previously had a kidney transplant (10.3\% epoetin, 33.3\% darbepoetin $\alpha, 56.3 \%$ CERA). Median [p25, p75] patient-month cost for patients with kidney transplant vs. patients who had not had a kidney transplant was: epoetin (191.3 [95.6, 414.5] euros vs. 103.2 [63.8, 191.3] euros, $p=0.060)$, darbepoetin $\alpha(144.0[72.0,288.0]$ euros vs. 144.0
$[72.0,216.0]$ euros, $p=0.136)$ and CERA $(196.7$ [172.1, 295.0] euros vs. $98.3[59.0,147.5]$ euros, $\mathrm{p}<0.001)$.

$30.9 \%$ patients were on dialysis $(35.0 \%$ epoetin, $58.3 \%$ darbepoetin $\alpha, 6.8 \%$ CERA). Median [p25, p75] patient-month cost for patients on dialysis vs. not yet on dialysis was: epoetin (151.1 [74.1, 239.1] euros vs. $92.1[59.5,165.6]$ euros, $p=0.006)$, darbepoetin $\alpha$ (144.0 [72.0, 216.0] euros vs. 144.0 [67.2, 229.2] euros, $p=0.888)$ and CERA (393.4 [98.3, 491.7] euros vs. 147.5 [98.3, 196.7] euros, $\mathrm{p}=0.035$ )

Conclusions The cost of epoetin and CERA is greater for both patients with a kidney transplant and patients on dialysis. However there was no difference regarding darbepoetin $\alpha$.

No conflict of interest.

\section{DGI-044 INHALED COLISTIN IN TREATMENT OF CHRONIC COLONISATION PSEUDOMONAS AERUGINOSA IN PATIENTS WITH NON-CYSTIC FIBROSIS BRONCHIECTASIS OR CHRONIC OBSTRUCTIVE PULMONARY DISEASE}

doi:10.1136/ejhpharm-2013-000276.310

B López Virtanen, S Vázquez Troche, J Valdueza Beneitez, B Nogal Fernández M Noguerol Cal, M Rodríguez María. Hospital El Bierzo, Pharmacy, Ponferrada, Spain

Background Chronic bronchial infection with Pseudomonas aeruginosa in patients with non-cystic fibrosis (CF) bronchiectasis/ chronic obstructive pulmonary disease (COPD) is related to worsening lung function and increased morbidity and mortality. Inhaled antibiotics represent an effective therapeutic approach for these diseases.

Purpose To evaluate the use of inhaled colistin in the treatment of chronic colonisation with Pseudomonas aeruginosa in patients with non-CF bronchiectasis/COPD.

Materials and Methods Retrospective study of patients with $\mathrm{COPD} /$ non-CF bronchiectasis colonised with Pseudomonas aeruginosa treated with inhaled colistin for at least three months from January 2008 to April 2012. Data collected: sex, age, diagnosis, duration of the treatment, disease-related hospitalizations pre and post-treatment, sputum cultures, clinical evolution.

Results 5 patients ( 3 with non-CF bronchiectasis and 2 with COPD) and 6 treatment episodes ( 1 patient received 2 courses of treatment) were included. Treatment duration was 27.6 months (range 4-48). Average cost per patient $€ 13,896$ (range $€ 2,950$ $25,888)$. In 5 episodes, treatment was initiated after $\geq 4$ consecutive sputum cultures positive for Pseudomonas resistant to tobramycin/ciprofloxacin. No difference in number of diseaserelated hospitalizations/month pre-and post-treatment ( 0.25 vs. 0.26). Sputum Pseudomonas eradication (3 consecutive negative sputum samples) was reported in 2 patients; treatment was continued, which was an unnecessary cost of $€ 15,500$ (22\% of total costs). No resistance developed to colistin. In two episodes (one with eradication) clinical improvement occurred (reduction in cough and expectoration).

The number of hospitalizations/month was similar before and after treatment, and the microbiological response (negative results on sputum) and the clinical response (reducing cough and sputum purulence) was moderate (2 of 6 episodes).

Three patients died from their bronchial disease.

Conclusions In most episodes the initial prescription was correct ( $\geq 3$ consecutive sputum cultures positive).

In patients whose Pseudomonas had been eradicated, treatment was continued, therefore sputum cultures should be monitored more frequently.

No effective treatment was observed.

No conflict of interest. 